\section{Avaliação das ações de planejamento em saúde empreendidas por equipes de saúde da família}

\author{
Evaluation of health planning activities \\ by family health teams
}

\author{
1 Centro de Ciências da \\ Saúde, Universidade Federal \\ do Espírito Santo, Vitória, \\ Brasil. \\ 2 Faculdade de Medicina, \\ Universidade Federal do Rio \\ de Janeiro, Rio de Janeiro, \\ Brasil. \\ 3 Programa de Pós-graduação \\ em Saúde Coletiva \\ Universidade Federal do \\ Espírito Santos, Vitória, \\ Brasil. \\ 4 Programa de Interação \\ Serviço, Ensino e \\ Comunidade, Centro \\ Universitário Vila Velha \\ Vila Velha, Brasil. \\ Correspondência \\ T. D. Sarti \\ Departamento de Medicina \\ Social, Centro de Ciências da \\ Saúde, Universidade Federal \\ do Espírito Santo. \\ Av. Marechal Campos 1468, \\ Vitória, ES 29090-041, Brasil. \\ thiagosarti@yahoo.com.br
}

\begin{abstract}
Evaluation of health planning activities adapted to the local context is an essential part of daily work in the Family Health Strategy (FHS). This study aimed to evaluate the planning activities conducted by family health teams in Espirito Santo State, Brazil. This was an ecological study based on secondary data from 241 health teams in 46 municipalities (counties) in the State that use the Evaluation for Quality Improvement in the Family Health Strategy (AMQ-ESF) form. Criteria were set for evaluating the responses by health professionals to the quality standards in the AMQ-ESF forms, and health planning indicators were listed. The responses were stratified according to the municipality's population size and time since implementation of the FHS and population coverage. The study mostly showed low adequacy rates in the teams' work processes in relation to the AMQ quality standards, and stratification of the variables had no influence on the local planning practices. Specific measures are needed to upgrade the work processes in the FHS.
\end{abstract}

Family Health; Health Planning; Health Evaluation; Quality of Health Care

\author{
Thiago Dias Sarti 1 \\ Carlos Eduardo Aguilera Campos 2 \\ Eliana Zandonade 3 \\ Gustavo Enrico Cabral Ruschi 4 \\ Ethel Leonor Noia Maciel 3
}

\section{Introdução}

A Estratégia Saúde da Família (ESF) é o principal modelo de organização da atenção primária à saúde no Brasil ${ }^{1}$. Em 1994, foi criada como programa focal de atenção básica a populações marginalizadas do Nordeste brasileiro, sendo que em 1998 foi reformulada no sentido de ser a estratégia de reorganização da atenção primária à saúde e de todo o sistema público de saúde 2,3,4. Em linhas gerais, a reorientação de modelo tecnoassistencial proposta pela ESF caracteriza-se pelo deslocamento do eixo de ação do indivíduo e sua doença para o cuidado integral de pessoas tomadas em seu contexto familiar e comunitário, tendo como suporte teórico e prático a integralidade da atenção, a promoção da saúde e a vigilância em saúde 1 .

Neste sentido, os serviços de saúde da família devem se organizar de tal forma que contemplem o cuidado às demandas da população e a outras necessidades de saúde não percebidas, como o rastreio de doenças e educação em saúde 5. Cuidados dirigidos a uma população específica incluem, além da atenção ao indivíduo, a vigilância dos problemas mais importantes e seus determinantes, o planejamento das intervenções preventivas e terapêuticas mais efetivas para a população e um movimento para a melhoria dos níveis de saúde e das condições de vida das pessoas 6,7. Assim, adentrar o cotidiano 
da população requer dos profissionais de saúde da ESF um olhar qualificado para a identificação destes problemas e exige a utilização de um conjunto de tecnologias adequadas para se lidar com eles.

Os avanços obtidos com a implantação e expansão da ESF nos municípios brasileiros são muitos, incluindo a expansão do acesso da população aos serviços de saúde 8,9, a promoção da equidade em saúde 10,11,12, a redução das taxas de mortalidade infantil 10,13,14 e de internações por condições sensíveis à atenção primária à saúde 15 , uma melhor avaliação da ESF por parte dos usuários quando comparada com serviços tradicionais de atenção primária à saúde 16,17, a melhoria do cuidado a diversas condições de saúde 12,18,19,20 e uma maior participação da população na gestão dos serviços de saúde 21,22.

Contudo, estudos mostram que, apesar dos avanços, a participação da população no cotidiano dos serviços pouco tem influenciado na sua organização e na formulação de projetos e ações atrelados às necessidades da comunidade 23,24. Outros autores encontraram uma carência de iniciativas para o planejamento das ações de saúde, resultando em um processo de trabalho parcelar, pouco reflexivo e ancorado na resolução de problemas imediatos surgidos principalmente do contato da equipe com os usuários que demandam por atendimentos clínicos não programados 25,26,27,28.

Por outro lado, há evidências que mostram dificuldades no trabalho em equipe no âmbito da ESF tanto na interação dos profissionais entre si e com os usuários quanto na integração das ações 29,30; no estabelecimento de ações que incidam sobre os principais problemas de saúde da população adscrita 23,28; na utilização de informações para o planejamento, oriundas do Sistema de Informação da Atenção Básica (SIAB) e outros sistemas de informação ${ }^{31}$; no estabelecimento de vínculos com a população 32 ; e na articulação de intervenções intersetoriais, que em geral são incipientes ${ }^{33}$. Ou seja, ocorre a persistência de um modo de organizar serviços e processos de trabalho que se distancia do ideário da ESF, embora mudanças concretas tenham sido sinalizadas no decorrer de sua implementação no país.

No entanto, poucos trabalhos se destinaram a avaliar a ESF no que toca à utilização de dispositivos e processos específicos de planejamento pelas equipes de saúde, sendo que não há estudos com tal recorte que utilize indicadores de processo dispostos na Avaliação para a Melhoria da Qualidade da Estratégia Saúde da Família (AMQ-ESF). É importante salientar que, embora existam outros instrumentos de avalia- ção da atenção primária à saúde, a escolha pelo AMQ-ESF se deu por este ser um instrumento desenvolvido e validado para utilização na ESF de forma a possibilitar a análise de processos de trabalho específicos do modelo brasileiro, bem como pela já apontada carência de estudos com este desenho. Diante do exposto, o objetivo deste estudo foi avaliar a implementação de ações e ferramentas essenciais para o planejamento local pelas equipes de saúde da família do Espírito Santo, Brasil, com base nos indicadores do AMQ-ESF.

\section{Metodologia}

Trata-se de um estudo ecológico, retrospectivo de análise de dados secundários, onde foram utilizados os dados disponíveis de 46 dos 64 municípios que aderiram ao processo AMQ-ESF no Espírito Santo até dezembro de 2007. Esta data refere-se ao fechamento do primeiro momento avaliativo do AMQ-ESF realizado pelos municípios estudados, tal como preconizado pelo Ministério da Saúde, sendo, portanto, a linha de base avaliativa da ESF nos locais estudados. O Espírito Santo é o estado da Federação com o maior grau de adesão e utilização da AMQ-ESF no país, sendo que $48 \%$ ( $n=241$ ) das equipes de saúde da família implantadas em 2007 neste estado responderam os questionários de autoavaliação.

O projeto AMQ-ESF foi lançado pelo Ministério da Saúde em 2005, como parte da Política Nacional de Avaliação da Atenção Básica, consistindo de cinco cadernos de autoavaliação normativa e participativa da ESF. Estes cadernos são formados por padrões de qualidade destinados a verificar a adequação das estruturas e processos de trabalho de gestores e profissionais das equipes de saúde vinculados à ESF, de forma a fornecer aos atores locais uma descrição pormenorizada do que se considera desejável para uma atenção primária à saúde ${ }^{34}$. Os padrões são variáveis categóricas nominais dicotômicas de resposta "sim" para as estruturas e processos adequadamente implantados e "não" no caso de implementação incompleta ou inexistente, sendo que os mesmos são acompanhados por orientações básicas que padronizam o processo de resposta dos gestores e profissionais a respeito do que está sendo avaliado 34 .

O conteúdo dos cadernos que compõem o AMQ-ESF foi desenvolvido por um painel de especialistas coordenado pelo Ministério da Saúde no ano de 2004. Este painel agregava as diversas áreas técnicas do Ministério da Saúde que tinham alguma interface com a ESF, bem como representantes da Organização Pan-Americana 
da Saúde (OPAS), Banco Mundial, Universidade Federal do Rio de Janeiro (UFRJ), Conselho Nacional de Secretários de Saúde (CONASS), Conselho Nacional de Secretarias Municipais de Saúde (CONASEMS) e Secretarias Estaduais de Saúde da Bahia, Ceará, Espírito Santo e Minas Gerais, sendo que o pré-teste do produto deste trabalho foi realizado nos municípios de Olinda (Pernambuco) e Petrópolis (Rio de Janeiro). Os critérios para a seleção destes municípios foram a facilidade de negociação das etapas do préteste com os gestores e profissionais e a possibilidade de se aplicar os instrumentos em realidades distintas de implantação da ESF (Nordeste e Sudeste do país). A partir das análises deste momento, procedeu-se à revisão dos padrões de qualidade e sua distribuição inicial entre os cadernos de autoavaliação 34 .

A validação dos instrumentos ocorreu por meio de um trabalho de campo que envolveu gestores e cerca de 500 equipes de saúde da família distribuídas em 25 municípios pertencentes a 21 estados da Federação. Os critérios utilizados para a seleção destes municípios foram: tempo de implantação da ESF (< 1 ano; entre 1 e 3 anos; entre 5 e 7 anos; e 10 anos ou mais); cobertura populacional da ESF (<30\%; entre $30 \%$ e $50 \%$; entre $50 \%$ e $70 \%$; e > 70\%); porte populacional do município (<20 mil habitantes; entre 20 e 50 mil; entre 50 e 100 mil; e > 100 mil habitantes); representatividade regional (municípios das cinco regiões do país); continuidade da gestão municipal em relação à eleição de 2002 (manutenção e não manutenção da gestão); existência e modalidade de equipes de saúde bucal (municípios com e sem equipes de saúde bucal, contemplando suas duas modalidades); e concordância com a participação no processo. Desta forma, buscou-se contemplar as várias possibilidades de implantação da ESF de forma a garantir a validade externa dos instrumentos 34 .

Em cada município eleito para participar da validação do AMQ-ESF, um coordenador local era designado e capacitado, sendo este o responsável por todas as fases de aplicação dos instrumentos com os gestores e profissionais. A seleção das equipes de saúde era feita de forma aleatória, respeitando o quantitativo estabelecido no protocolo de pesquisa calculado com base no número total de equipes de saúde da família do município: até 19 equipes - 100\% participariam da validação; 20 a 39 equipes $-80 \%$; 40 a 69 equipes $-60 \%$; 70 a 99 equipes $-40 \%$; acima de 100 equipes $-25 \%$. Os secretários de saúde, os coordenadores da atenção primária e os gestores das unidades de saúde às quais pertenciam as equipes também participaram da validação dos respectivos instrumentos 34 .
O coordenador local do processo de validação do AMQ-ESF organizava reuniões com os atores selecionados, sendo que nestes encontros os instrumentos de autoavaliação eram preenchidos e avaliados quanto a sua clareza, pertinência e validade interna por meio de um questionário específico para este fim. As críticas e sugestões eram registradas pelo coordenador e todo o material proveniente das reuniões era encaminhado ao Ministério da Saúde para análise e categorização. Após este momento, um membro do painel de especialistas visitava o município com o intuito de avaliar todo o processo de distribuição dos questionários, coleta de dados e armazenamento e transmissão do material. Estas informações foram então utilizadas para modificar os instrumentos de autoavaliação e consolidar os cinco cadernos que compõem a versão final do AMQ-ESF 34.

A metodologia utilizada para o preenchimento dos instrumentos de autoavaliação no processo de validação do AMQ-ESF foi mantida como orientação básica a partir de sua implantação oficial. Os padrões de qualidade são apresentados com uma descrição básica do que se almeja avaliar em termos de estrutura e processos de trabalho na ESF, estando em anexo um detalhamento do que é necessário considerar para que se responda afirmativamente ao mesmo. A ideia geral do AMQ-ESF é apontar âmbitos da gestão e do cotidiano das equipes que são passíveis de melhorias. Sendo assim, os atores são orientados a responder "sim" apenas no caso de cumprir com todos os critérios apontados no anexo de cada padrão de qualidade. Em caso de implantação incompleta de algum processo de trabalho ou estrutura, a resposta deveria ser "não", possibilitando o surgimento de um processo reflexivo e a respeito destas insuficiências. Desta forma, assegura-se a homogeneidade dos procedimentos de coleta de dados. Além disso, como a equipe, a coordenação do serviço e a gestão do sistema local de saúde preenchem apenas um questionário para cada um destes níveis de prática, a resposta final para cada padrão de qualidade era obtida por consenso 34 .

No presente trabalho, para se avaliar o grau de implantação de estruturas e processos relacionados ao planejamento local em saúde, utilizou-se dos padrões do caderno 4 de autoavaliação do AMQ-ESF, que é aquele destinado à autoavaliação das equipes de saúde da família, que estivessem relacionados a algumas das etapas gerais de um processo de planejamento: identificar e descrever os problemas; propor mudanças e definir as intervenções; e avaliar os resultados das intervenções 5,35. A análise dos dados foi feita por meio de uma estatística descritiva composta de 
média de percentual de respostas "sim" aos padrões de qualidade e desvio padrão, sendo que a unidade de análise foi o agrupamento das equipes de saúde por município estudado. Para tal, utilizou-se o pacote estatístico SPSS 15.0 (SPSS Inc., Chicago, Estados Unidos) após reorganização das planilhas a partir do banco original de dados disponibilizado pela Coordenação da Avaliação e Acompanhamento do Departamento de Atenção Básica do Ministério da Saúde.

Um dos aspectos fundamentais do AMQESF é a lógica incremental de seus padrões de qualidade ${ }^{34}$. São descritos cinco estágios de qualidade, nos quais são dispostos os padrões de qualidade de acordo com sua complexidade teórica de implementação. Definiram-se pontos de corte para análise das médias de percentual de respostas "sim" aos padrões de qualidade de acordo com os estágios de qualidade definidos pelo AMQ-ESF. Desta forma, o critério de análise das variáveis quanto a sua adequação de implantação será: estágio elementar (E) - 80\% ou mais; estágio desenvolvimento (D) - $60 \%$ a $79,9 \%$; estágio consolidado (C) $-40 \%$ a $59,9 \%$; estágio bom - $20 \%$ a $39,9 \%$; e estágio avançado (A) $-10 \%$ a $19,9 \%$. 36 .

Também se realizou uma análise estratificada dos padrões de qualidade selecionados a partir de características dos municípios componentes da amostra. Para tal, utilizaram-se cortes relacionados ao tempo de implantação da ESF no município, à cobertura populacional da ESF e ao porte populacional do município 36 , conforme descrito na Tabela 1. Para a análise estratificada para tempo de implantação da ESF e porte populacional do município, utilizou-se o teste estatístico t com nível de 5\% de significância e para a análise estratificada com relação à cobertura populacio- nal da ESF, utilizou-se o teste ANOVA e o teste $a$ posteriori de Duncan (Duncan's post-hoc test), a um nível de $5 \%$ de significância. A hipótese de trabalho nesta análise estratificada é o grau de implantação dos padrões de qualidade preconizados pelo AMQ-ESF ser diretamente proporcional ao tempo de implantação e cobertura populacional da ESF e inversamente proporcional ao porte populacional do município.

Todas as diretrizes e normas regulamentadoras de pesquisas envolvendo seres humanos previstas na Declaração de Helsinki e na Resolução $n^{\circ}$. 196/96 da Comissão Nacional de Ética em Pesquisa (CONEP) foram rigorosamente seguidas, sendo o trabalho aprovado em Comitê de Ética em Pesquisa (CEP) da Universidade Federal do Espírito Santo (UFES) (com o parecer nº. 035/08). Um requisito do AMQ-ESF para utilização dos dados é o sigilo quanto à identidade de todos os participantes do processo de avaliação, o qual foi seguido irrestritamente no desenvolvimento desta pesquisa.

\section{Resultados}

A distribuição dos municípios pertencentes à amostra em termos populacionais está descrita na Tabela 2. Observa-se que a amostra, quando comparada com o perfil dos municípios do Espírito Santo, possui um percentual menor de municípios com até 20 mil habitantes. Outras características destes municípios é que no geral o Índice de Desenvolvimento Humano (IDH) destes está abaixo da média estadual, assim como a taxa de urbanização, por serem municípios pequenos do interior do estado.

Tabela 1

Critérios de avaliação dos padrões de resposta das equipes de saúde da família à Avaliação para a Melhoria da Qualidade da Estratégia Saúde da Família (AMQ-ESF). Espírito Santo, Brasil, 200712.

\begin{tabular}{|c|c|c|}
\hline Variável & Estratificação & Critérios \\
\hline \multirow[t]{3}{*}{ Tempo de Implantação da ESF } & 1 & 2 anos \\
\hline & 2 & 4 anos \\
\hline & 3 & 6 anos \\
\hline \multirow[t]{4}{*}{ Cobertura populacional } & 4 & $\mathrm{P} 25=44 \%$ de cobertura \\
\hline & 5 & P50 = 95\% de cobertura \\
\hline & 6 & P75 $=100 \%$ de cobertura \\
\hline & 7 & $<\mathrm{P} 25 / \mathrm{P} 25-\mathrm{P} 50 />\mathrm{P} 50$ \\
\hline \multirow[t]{2}{*}{ Porte populacional } & 8 & $<100$ mil habitantes/ \\
\hline & & $\geq 100$ mil habitantes \\
\hline
\end{tabular}


Estratificação dos municípios conforme tamanho da população. Espírito Santo, Brasil, 2007.

\begin{tabular}{lcccc}
\hline & \multicolumn{2}{c}{ Amostra } & & Espírito Santo \\
Tamanho populacional & $\mathbf{n}$ & $\%$ & $\mathbf{n}$ & 60,26 \\
\hline Até 20 mil habitantes & 24 & 52,17 & 47 & 29,49 \\
$20-100$ mil habitantes & 14 & 30,43 & 23 & 10,26 \\
Superior a 100 mil habitantes & 8 & 17,39 & 8 & 100,0 \\
Total & 46 & 100,0 & 78 & 1 \\
\hline
\end{tabular}

Os padrões de qualidade utilizados como variáveis neste estudo, seus respectivos estágios de qualidade na estratégia AMQ e suas médias de percentual de respostas "sim" estão dispostos na Tabela 3. No geral, as ações de planejamento e programação em saúde elencadas neste trabalho se mostraram parcialmente implementadas nas equipes de saúde da família estudadas.

Com relação à etapa "identificar e descrever os problemas”, algumas potencialidades e limites das equipes de saúde foram encontrados. Quanto à organização das fontes de informação, a maioria das equipes $(72,2 \%)$ organiza seus prontuários por núcleo familiar, embora um percentual menor das equipes $(65,7 \%)$ atualiza os cadastros familiares mensalmente. Quanto ao diagnóstico da situação de saúde da população, 42,5\% das equipes o realizam e $36,1 \%$ possuem registros de aspectos variados do território (aspectos demográficos, socioeconômicos, étnicos, culturais, ambientais e sanitários da área adscrita).

Ainda nesta etapa, 55,8\% das equipes trabalham com mapa de sua área de abrangência, 21,4\% identificam situações dinâmicas do território e $11,8 \%$ organizam painéis de situação. Quanto ao monitoramento dos processos de trabalho, $28,1 \%$ das equipes monitoram as referências para outros níveis de atenção e $36,3 \%$ as solicitações de exames diagnósticos. Os percentuais de equipes que monitoram o tipo de solicitação pelo serviço (por exemplo, consultas de crianças, mulheres, hipertensos, idosos, exames e procedimentos, comparando-os com a demanda total pelas ações da equipe) e o tempo médio de espera por estes são, respectivamente, $33,2 \%$ e $36,3 \%$.

Na etapa "propor mudanças e definir as intervenções”, observou-se que $57,8 \%$ das equipes utilizam o SIAB para planejar suas ações e $28,5 \%$ das equipes diagnosticam os problemas, planejam as intervenções e realizam as ações de maneira integrada. Por outro lado, observou-se que $26,2 \%$ das equipes, planejam, executam e acom- panham as ações na sua área de atuação em parceria e/ou articulação informal com ONG, associações, conselhos, igrejas e movimentos sociais. Contudo, apenas 8,4\% das equipes elaboram estratégias para o enfrentamento dos principais problemas sociais de seus territórios adscritos e $8,5 \%$ inserem representantes de movimentos sociais em seu planejamento cotidiano de forma a ampliar a compreensão das necessidades de saúde da população, ambos abaixo do esperado.

Por último, na etapa "avaliar o resultado das intervenções”, observa-se que $42,6 \%$ das equipes realizam reuniões semanais com todos os seus membros para discutir casos, resolver conflitos, planejar as ações e compartilhar saberes, 18,5\% avaliam suas ações ao menos semestralmente e $24,3 \%$ delas participam da maior parte das reuniões do conselho municipal de saúde. Entretanto, $38,5 \%$ das equipes realizam avaliações mais frequentes, ao menos mensal, e planejam a continuidade de suas ações; $24,5 \%$ das equipes disponibilizam recursos para registro de sugestões e reclamações dos usuários; e 7,1\% reúnem-se com a comunidade trimestralmente para debater os problemas locais de saúde, a assistência prestada e os resultados alcançados. Quanto à análise estratificada, observa-se que alguns padrões de qualidade alcançaram significância estatística em alguns estratos, conforme pode ser visualizado na Tabela 4 .

\section{Discussão}

Os resultados deste trabalho mostram uma heterogeneidade e uma incompletude na implantação de processos de trabalho e ferramentas de planejamento pelas equipes de saúde da família dos municípios do Espírito Santo. Como potencialidades, essas equipes organizam o registro clínico por núcleo familiar e dispõem de mapas do território com informações abrangentes e relevantes, monitoram alguns processos e fluxos de 
Ações de planejamento local em saúde na Estratégia Saúde da Família (ESF). Espírito Santo, Brasil, 2007.

\begin{tabular}{|c|c|c|c|c|}
\hline $\begin{array}{l}\text { Número do padrão de } \\
\text { qualidade }\end{array}$ & Estágio de qualidade * & Descrição do padrão de qualidade & Média ** & DP \\
\hline \multicolumn{5}{|l|}{$\begin{array}{l}\text { Identificar e descrever os } \\
\text { problemas }\end{array}$} \\
\hline 4.1 & $E(80,0 \%-100,0 \%)$ & O cadastramento das famílias é atualizado mensalmente. & 65,7 & 39,0 \\
\hline 4.9 & $D(60,0 \%-79,9 \%)$ & $\begin{array}{l}\text { Os prontuários estão organizados por núcleos familiares, } \\
\text { fortalecendo o modelo de atenção da saúde da família. }\end{array}$ & 72,2 & 33,6 \\
\hline 4.7 & $D(60,0 \%-79,9 \%)$ & $\begin{array}{l}\text { A equipe realiza diagnóstico da situação de saúde da } \\
\text { população, identificando os problemas mais frequentes. }\end{array}$ & 42,5 & 34,5 \\
\hline 4.6 & $D(60,0 \%-79,9 \%)$ & $\begin{array}{l}\text { A ESF trabalha com mapa da sua área de atuação no qual estão } \\
\text { discriminadas as microáreas de responsabilidade dos agentes } \\
\text { comunitários de saúde. }\end{array}$ & 55,8 & 33,9 \\
\hline 4.13 & C $(40,0 \%-59,9 \%)$ & $\begin{array}{c}\text { A ESF registra e monitora as solicitações de exames } \\
\text { diagnósticos. }\end{array}$ & 36,3 & 34,6 \\
\hline 4.17 & B $(20,0 \%-39,9 \%)$ & $\begin{array}{l}\text { A ESF monitora a procura pelo serviço quanto ao tipo de } \\
\text { solicitação, verificando o percentual de atendimento. }\end{array}$ & 33,2 & 31,6 \\
\hline 4.31 & B $(20,0 \%-39,9 \%)$ & $\begin{array}{l}\text { Existe monitoramento do tempo médio de espera pelos } \\
\text { serviços. }\end{array}$ & 36,3 & 31,9 \\
\hline 4.18 & B $(20,0 \%-39,9 \%)$ & $\begin{array}{l}\text { Os mapas de trabalho da equipe estão atualizados e apontam } \\
\text { situações dinâmicas do território e sua população. }\end{array}$ & 21,4 & 29,5 \\
\hline 4.20 & $A(10,0 \%-19,9 \%)$ & $\begin{array}{c}\text { A ESF organiza "painel de situação" com os mapas, dados e } \\
\text { informações de saúde do território. }\end{array}$ & 11,8 & 19,9 \\
\hline 4.19 & B $(20,0 \%-39,9 \%)$ & $\begin{array}{l}\text { A ESF trabalha o diagnóstico, o planejamento e a realização das } \\
\text { ações para o território de maneira integrada. }\end{array}$ & 28,5 & 30,0 \\
\hline 4.48 & B $(20,0 \%-39,9 \%)$ & $\begin{array}{l}\text { A equipe planeja, executa e acompanha as ações na sua área } \\
\text { de atuação em parceria e/ou articulação informal com ONG, } \\
\text { associações, conselhos, igrejas e movimentos sociais. }\end{array}$ & 26,2 & 26,1 \\
\hline 4.58 & $A(10,0 \%-19,9 \%)$ & $\begin{array}{l}\text { Existe participação de representantes de movimentos sociais e } \\
\text { usuários no processo de planejamento do trabalho das ESF. }\end{array}$ & 8,5 & 18,0 \\
\hline
\end{tabular}

(continua) 
Tabela 3 (continuação)

\begin{tabular}{|c|c|c|c|c|}
\hline $\begin{array}{l}\text { Número do padrão de } \\
\text { qualidade }\end{array}$ & Estágio de qualidade * & Descrição do padrão de qualidade & Média ** & DP \\
\hline \multicolumn{5}{|l|}{$\begin{array}{l}\text { Avaliar o resultado das } \\
\text { intervenções }\end{array}$} \\
\hline 4.26 & $D(60,0 \%-79,9 \%)$ & $\begin{array}{l}\text { Recursos para registro de sugestões e reclamações estão ao } \\
\qquad \text { alcance dos usuários. }\end{array}$ & 24,5 & 32,6 \\
\hline 4.16 & $C(40,0 \%-59,9 \%)$ & $\begin{array}{l}\text { A ESF dedica uma reunião mensal à avaliação dos resultados } \\
\text { alcançados e planejamento da continuidade das ações. }\end{array}$ & 38,5 & 31,9 \\
\hline 4.56 & $C(40,0 \%-59,9 \%)$ & $\begin{array}{c}\text { A ESF reúne-se com a comunidade trimestralmente para } \\
\text { debater os problemas locais de saúde, a assistência prestada e } \\
\text { os resultados alcançados. }\end{array}$ & 7,1 & 18,8 \\
\hline 4.15 & $C(40,0 \%-59,9 \%)$ & A ESF dedica um período da semana para reunião de equipe. & 42,6 & 39,1 \\
\hline 4.57 & B $(20,0 \%-39,9 \%)$ & A ESF participa de reuniões com conselhos de saúde. & 24,3 & 28,1 \\
\hline 4.21 & $A(10,0 \%-19,9 \%)$ & A ESF realiza avaliação semestral dos resultados alcançados. & 18,5 & 29,2 \\
\hline
\end{tabular}

DP: desvio-padrão; SIAB: Sistema de Informação da Atenção Básica.

* As letras referem-se ao estágio de qualidade ao qual o padrão pertence: E: elementar; D: desenvolvimento; C: consolidado; B: bom; A: avançado; ** Média de percentual de respostas "sim" aos padrões de qualidade do projeto Avaliação para a Melhoria da Qualidade da Estratégia Saúde da Família (AMQ-ESF).

Padrões de resposta das equipes de saúde da família à Avaliação para a Melhoria da Qualidade da Estratégia Saúde da Família (AMQ-ESF) com significância estatística, estratificados por tempo de implantação e cobertura populacional da ESF e porte populacional do município. Espírito Santo, Brasil, 2007.

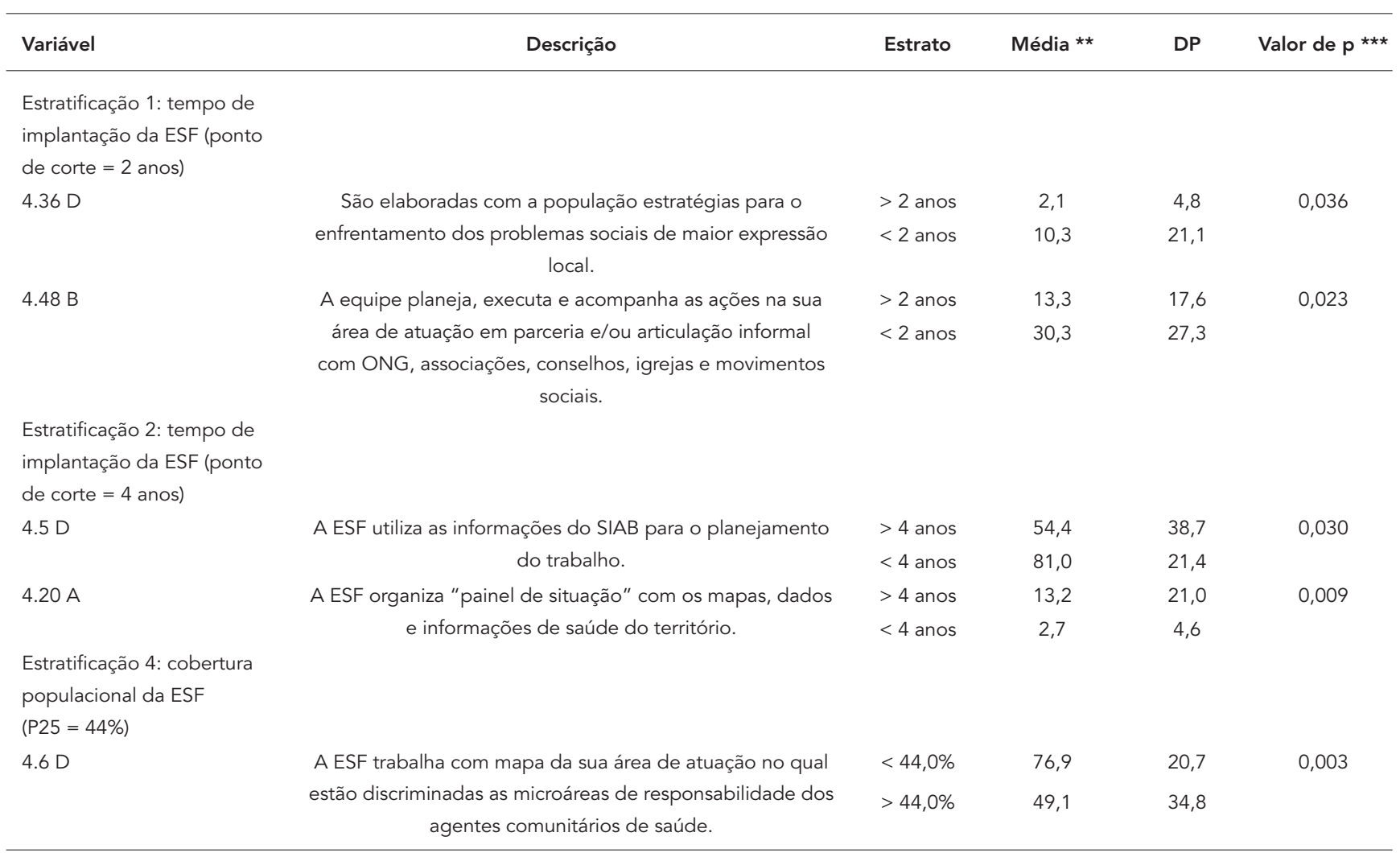

(continua) 
Tabela 4 (continuação)

\begin{tabular}{|c|c|c|c|c|c|}
\hline Variável & Descrição & Estrato & Média ** & DP & Valor de $p * \star \star$ \\
\hline \multirow[t]{2}{*}{$4.20 \mathrm{~A}$} & A ESF organiza "painel de situação" com os mapas, dados & $<44,0 \%$ & 3,8 & 7,9 & 0,020 \\
\hline & e informações de saúde do território.. & $>44,0 \%$ & 14,3 & 21,9 & \\
\hline \multirow[t]{2}{*}{$4.21 \mathrm{~A}$} & A ESF realiza avaliação semestral dos resultados & $<44,0 \%$ & 5,0 & 9,2 & 0,005 \\
\hline & alcançados. & $>44,0 \%$ & 22,7 & 32,0 & \\
\hline \multicolumn{6}{|c|}{ Estratificação 7: cobertura } \\
\hline \multicolumn{6}{|c|}{ populacional da ESF } \\
\hline \multicolumn{6}{|c|}{$(<\mathrm{P} 25 / \mathrm{P} 25-\mathrm{P} 50 />\mathrm{P} 50)$} \\
\hline \multirow[t]{3}{*}{$4.6 \mathrm{D}$} & A ESF trabalha com mapa da sua área de atuação no qual & $<\mathrm{P} 25$ & 79,7 & 21,4 & 0,040 \\
\hline & estão discriminadas as microáreas de responsabilidade dos & P25-P50 & 75,2 & 20,2 & \\
\hline & agentes comunitários de saúde. & $>$ P50 & 48,4 & 35,0 & \\
\hline \multirow[t]{3}{*}{$4.14 \mathrm{C}$} & A ESF notifica os usuários sobre a marcação de consultas & $<\mathrm{P} 25$ & 68,9 & 46,3 & 0,019 \\
\hline & especializadas e ou exames. & P25-P50 & 80,2 & 21,0 & \\
\hline & & $>$ P50 & 93,9 & 13,1 & \\
\hline \multirow[t]{3}{*}{ 4.19 D } & A ESF trabalha o diagnóstico, o planejamento e a & $<$ P25 & 33,6 & 22,8 & 0,030 \\
\hline & realização das ações para o território de maneira & P25-P50 & 52,5 & 34,4 & \\
\hline & integrada. & $>\mathrm{P} 50$ & 22,3 & 27,3 & \\
\hline \multicolumn{6}{|c|}{ Estratificação 8: porte } \\
\hline \multicolumn{6}{|c|}{ populacional do município } \\
\hline \multirow[t]{4}{*}{$4.13 \mathrm{C}$} & A ESF registra e monitora as solicitações de exames & $+100 \mathrm{mil}$ & 15,86 & 17,17 & 0,007 \\
\hline & diagnósticos. & habitantes & & & \\
\hline & & $-100 \mathrm{mil}$ & 40,65 & 35,87 & \\
\hline & & habitantes & & & \\
\hline \multirow[t]{4}{*}{$4.21 \mathrm{~A}$} & A ESF realiza avaliação semestral dos resultados & $+100 \mathrm{mil}$ & 5,38 & 7,89 & 0,008 \\
\hline & alcançados. & habitantes & & & \\
\hline & & $-100 \mathrm{mil}$ & 21,27 & 31,26 & \\
\hline & & habitantes & & & \\
\hline
\end{tabular}

DP: desvio-padrão; SIAB: Sistema de Informação da Atenção Básica.

* As letras que seguem o número do padrão de qualidade referem-se ao estágio de qualidade ao qual o padrão pertence: E: elementar; D: desenvolvimento;

C: consolidado; B: bom; A: avançado;

** Média de percentual de respostas "sim" aos padrões de qualidade do AMQ-ESF;

*** Nível de significância para o teste t e ANOVA, quando pertinentes.

trabalho no serviço, realizam reuniões de equipe com frequência e, em certa medida, se articulam com os movimentos organizados da comunidade para o planejamento das ações.

Contudo, tomadas em sua totalidade, as médias de respostas "sim" aos padrões de qualidade selecionados do caderno 4 de autoavaliação do AMQ-ESF são baixas e mostram as dificuldades de se articularem ferramentas e ações coletivas para o planejamento e monitoramento das intervenções no contexto local. Quanto a isto, uma das questões a serem levantadas é a complexidade dos padrões de qualidade destinados à normatização das ações de diagnóstico local e planejamento das intervenções propostos pelo AMQ-ESF. O único padrão de qualidade selecionado que pertence ao estágio de qualidade elementar (E) é o que se refere à atualização mensal do cadastramento das famílias do território, e mesmo este padrão ficou com uma média abaixo do esperado.

Corroborando alguns resultados deste estudo, Kawata et al. 26 mostram que, contrário às características centrais da ESF enunciadas pelos documentos oficiais do Ministério da Saúde, as equipes de saúde raramente planejam suas ações em conjunto com a comunidade e outros trabalhadores dos serviços, sendo que as ações de planejamento são geralmente desenvolvidas de forma centralizada e pouco participativa. Por outro lado, tanto neste estudo como em Mishima 27, não foram identificados processos sistemáticos de avaliação e monitoramento das ações.

Também se pode aventar a resistência em se reorientar o modelo biomédico na atenção primária à saúde, ainda persistindo na ESF processos centrados em procedimentos e na atenção 
individual curativa 37 , sendo a formação acadêmica dos profissionais de saúde inadequada para este cenário assistencial um dos fatores relacionados. Um discurso muito comum é que a demanda por consultas individuais sufoca as equipes, deixando pouco tempo para a execução de outros processos de trabalho fundamentais na atenção primária à saúde, embora a resolução deste problema passe exatamente por um planejamento e programação dos processos de trabalho de forma adequada 25.

Além disso, esta realidade não sofre grande influência do tempo de implantação e cobertura populacional da ESF. Portanto, não se pode afirmar que um maior tempo de implantação e uma maior cobertura populacional da ESF indicariam uma maior maturidade municipal em termos de organização de seus sistemas locais de saúde e significariam uma maior probabilidade de adequação dos processos de trabalho das equipes de saúde aos padrões de qualidade do AMQ-ESF.

Quanto a isto, a adoção da saúde da família como estratégia prioritária para a organização da atenção básica municipal tem mostrado resultados conflitantes segundo alguns estudos. Elias et al. 16, Machado et al. 38 e Escorel et al. 39 encontraram que a prioridade dada à ESF está relacionada com uma melhor avaliação da atenção básica municipal, levando-se em consideração princípios como acessibilidade, cuidado com enfoque familiar e orientação comunitária. Por sua vez, Viana et al. ${ }^{9}$ mostram que a integralidade e o acesso universal estão relacionados com a centralidade da atenção básica no município. Contudo, Ibañez et al. 17, utilizando as mesmas categorias de análise da atenção primária à saúde para os municípios de São Paulo, não encontraram relação entre estes atributos e a proporção de produção ambulatorial municipal, estando aqueles mais relacionados com os indicadores sociais dos municípios.

Por outro lado, uma série de trabalhos vem mostrando o impacto da ESF em indicadores de mortalidade infantil, mostrando que maiores taxas de cobertura populacional e maior tempo de implantação estão relacionados a reduções significativas destes indicadores, principalmente nas regiões com piores indicadores socioeconômicos e de mortalidade infantil 10,13,14. Contudo, não há evidências consistentes que comprovem a relação entre o tempo de implantação e cobertura populacional da ESF e o processo de trabalho das equipes de saúde da família. E os resultados deste trabalho mostram que estas variáveis independentes não tiveram uma influência substancial nos processos autorreferidos de trabalho das equipes de saúde.
Quanto ao porte populacional dos municípios, também se observou pouca influência desta variável nos padrões de qualidade do AMQ-ESF, embora exista farta literatura mostrando as dificuldades dos grandes municípios em implantar eficazmente a ESF em seu território 40,41,42. Não é possível dizer se os aparatos institucionais de apoio às equipes - como educação permanente e gestão do trabalho, incluindo aí vínculos empregatícios - e a infraestrutura das unidades de saúde se diferenciam entre os municípios, o que poderia explicar possíveis diferenças entre o nível de implementação dos processos de trabalho das equipes de saúde. Contudo, parece haver relação, mesmo que mínima, entre processos de monitoramento e avaliação em favor de pequenos municípios, possivelmente explicados em decorrência da menor sobrecarga do sistema municipal com referências a outros níveis de atenção e com solicitações de exames propedêuticos. De qualquer forma o fraco desempenho das funções e de ações ligadas ao planejamento, observados no conjunto dos municípios indicam a necessidade de avaliar as falhas existentes nos processos de educação permanente das equipes e questionar a prioridade dada pelas instâncias gestoras a esta premissa básica da ESF.

É importante também frisar algumas limitações deste estudo. Um primeiro aspecto decorre de seu desenho metodológico. As principais limitações dos estudos ecológicos são a dificuldade de controlar os efeitos de potenciais fatores de confundimento, a multiplicidade de fontes de dados com qualidade variável e frequentemente não controlável e as dificuldades de se generalizar os resultados e as soluções encontradas para contextos locais distintos 43 . Outro ponto de relevância é o caráter autoaplicado e autoavaliativo do AMQ-ESF. As equipes de saúde respondem aos padrões de qualidade conforme seu próprio juízo de valor, a partir de discussões em grupo com vistas à obtenção de consenso na resposta que melhor atende ao que está preconizado no instrumento de autoavaliação, não sendo possível afirmar categoricamente que os processos indicados como sendo feitos são de fato praticados, pois não foram utilizadas técnicas de observação direta neste trabalho, sendo esta uma importante limitação do estudo.

Além disso, deve-se destacar a insuficiência dos procedimentos de validação do AMQ-ESF quando de sua proposição. Embora este seja o primeiro instrumento de avaliação da qualidade desenhado especificamente para a ESF, são necessários novos estudos que o comparem com outros instrumentos de avaliação da qualidade da atenção primária à saúde, estimando sua capacidade de mensurar e apontar problemas na 
organização da ESF. Da mesma forma, serão pertinentes análises que verifiquem a relação dos processos de trabalho elencados nos cadernos de autoavaliação com resultados em termos de melhoria da assistência prestada e da situação de saúde da população, bem como trabalhos que avaliem a concordância das respostas dadas pelas equipes a cada indicador com o que de fato é feito pelos profissionais. Neste sentido, os resultados deste trabalho relacionam-se à implantação de processos de planejamento local no cotidiano das equipes de saúde, conforme preconizado oficialmente pelos órgãos governamentais responsáveis pela condução das políticas de saúde no país, sendo importante que outros estudos problematizem estes processos e seu impacto na melhoria da qualidade da atenção primária à saúde e da saúde da população. De uma forma geral, estas limitações apontam para a necessidade de se garantir a realização de todos os procedimentos metodológicos pertinentes em

Resumo

Ações de planejamento em saúde adaptadas ao contexto local são fundamentais no cotidiano da Estratégia Saúde da Família (ESF). Este trabalho visa avaliar as práticas de planejamento empreendidas pelas equipes de saúde da família do Espírito Santo, Brasil. Trata-se de um estudo ecológico com análise dos dados secundários de 241 equipes de saúde de 46 municípios deste estado que utilizam a Avaliação para Melhoria da Qualidade da Estratégia Saúde da Família (AMQ-ESF). Foram estabelecidos critérios de avaliação das respostas dos profissionais de saúde aos padrões de qualidade dos instrumentos do AMQ-ESF e elencados os indicadores que versam sobre planejamento em saúde. As respostas foram estratificadas por porte populacional do município, tempo de implantação e cobertura populacional da ESF. Encontraram-se no geral baixas médias de adequação dos processos de trabalho das equipes aos padrões de qualidade do AMQ, sendo que não houve influência das variáveis de estratificação nas práticas de planejamento local. Ações específicas neste campo são necessárias para a qualificação do trabalho da ESF.

Saúde da Família; Planejamento em Saúde; Avaliação em Saúde; Qualidade da Assistência à Saúde qualquer processo de inovação em saúde, seja de iniciativa governamental, industrial ou acadêmica, como consta da Política Nacional de Ciência, Tecnologia e Inovação em Saúde 44.

Por fim, dada a carência de estudos que se utilizam de abordagens quantitativas para avaliar as ações de planejamento em saúde no contexto da ESF, os resultados aqui apresentados podem ser de grande utilidade para gestores, profissionais e acadêmicos, que de alguma forma estejam relacionados à atenção primária à saúde. Tendo em vista o grande número de equipes analisadas, conferindo uma boa representatividade de seus resultados, algumas potencialidades e fragilidades dos processos de trabalho das equipes de saúde da família que atuam nos municípios do Espírito Santo podem indicar a realidade da ESF no Brasil, exigindo esforços para a mudança desta realidade frente à importância da temática para a organização de serviços adequados para a população.

\section{Colaboradores}

T. D. Sarti, C. E. A. Campos, E. Zandonade, G. E. C. Ruschi e E. L. N. Maciel participaram da concepção e coordenação do estudo em todas as suas etapas, incluindo análise dos dados e redação do artigo.

\section{Agradecimentos}

Os autores agradecem a Iracema Benevides pelas contribuições feitas na descrição do processo de validação dos instrumentos de coleta de dados. 


\section{Referencias}

1. Ministério da Saúde. Política Nacional de Atenção Básica. Brasília: Ministério da Saúde; 2006.

2. Conill EM. Ensaio histórico-conceitual sobre a Atenção Primária à Saúde: desafios para a organização de serviços básicos e da Estratégia Saúde da Família em centros urbanos no Brasil. Cad Saúde Pública 2008; 24 Suppl 1:S7-16.

3. Ministério da Saúde. Programa de Saúde da Família. Brasília: Ministério da Saúde; 1994.

4. Ministério da Saúde. Saúde da família: uma estratégia para reorientação do modelo assistencial. Brasília: Ministério da Saúde; 1997.

5. Black N, Gruen R. Understanding health services. Berkshire: Open University Press; 2005.

6. van Weel C, Maeseneer J, Roberts R. Integration of personal and community health care. Lancet 2008; 372:871-2.

7. Lightburn A, Sessions P. Community-based clinical practice: re-creating the culture of care, In: Lightburn A, Sessions P, editors. Handbook of community-based clinical practice. New York: Oxford University Press; 2006. p. 19-38.

8. Ministério da Saúde. Saúde da família no Brasil: uma análise de indicadores selecionados, 19982004. Brasília: Ministério da Saúde; 2006.

9. Viana ALD, Rocha JSY, Elias PE, Ibanez N, Novaes MHD. Modelos de atenção básica nos grandes centros paulistas: efetividade, eficácia, sustentabilidade e governabilidade. Ciênc Saúde Coletiva 2006; 11:577-606

10. Aquino R, Oliveira NF, Barreto ML. Impact of the Family Health Program on infant mortality in Brazilian municipalities. Am J Public Health 2009; 99:87-93.

11. Goldbaum M, Gianini RJ, Novaes HMD, Cesar CLG. Utilização de serviços de saúde em áreas cobertas pelo programa saúde da família (Qualis) no Município de São Paulo. Rev Saúde Pública 2005; 39:90-9.

12. Harzheim E, Duncan BB, Stein AT, Cunha CR, Gonçalves MR, Trindade TG, et al. Quality and effectiveness of different approaches to primary care delivery in Brazil. BMC Health Serv Res 2006; 6:156.

13. Macinko J, Souza MFM, Guanais FC, Simões CCS. Going to scale with community-based primary care: an analysis of the family health program and infant mortality in Brazil, 1999-2004. Soc Sci Med 2007; 65:2070-80.

14. Macinko J, Guanais FC, Fátima M, Souza M. Evaluation of the impact of the Family Health Program on infant mortality in Brazil, 1990-2002. J Epidemiol Community Health 2006; 60:13-9.

15. Guanais F, Macinko J. Primary care and avoidable hospitalizations: evidence from Brazil. J Ambul Care Manage 2009; 32:115-22.

16. Elias PE, Ferreira CW, Alves MCG, Cohn A, Kishina $\mathrm{V}$, Escrivão Júnior $\mathrm{A}$, et al. Atenção básica em saúde: comparação entre PSF e UBS por estrato de exclusão social no município de São Paulo. Ciênc Saúde Coletiva 2006; 11:633-42.
17. Ibanez N, Rocha JSY, Castro PC, Ribeiro MCSA, Foster AC, Novaes MHD, et al. Avaliação do desempenho da atenção básica no Estado de São Paulo. Ciênc Saúde Coletiva 2006; 11:683-703.

18. Monteiro CA. Causas do declínio da desnutrição infantil no Brasil, 1996-2007. Rev Saúde Pública 2009; 43:35-43.

19. Xavier AJ, Reis SS, Paulo EM, d'Orsi E. Tempo de adesão à Estratégia de Saúde da Família protege idosos de eventos cardiovasculares e cerebrovasculares em Florianópolis, 2003 a 2007. Ciênc Saúde Coletiva 2008; 13:1543-51.

20. Gomes KO, Cotta RMM, Euclydes MP, Targueta CL, Priore SE, Franceschini SCC. Avaliação do impacto do Programa Saúde da Família no perfil epidemiológico da população rural de Airões, município de Paula Cândido (MG), 1992-2003. Ciênc Saúde Coletiva 2009; 14 Suppl 1:1473-82.

21. Lacerda WA, Santiago IMFL. A participação popular na gestão local do Programa Saúde da Família em Campina Grande, Paraíba. Revista Katálysis 2007; 10:197-205.

22. Marin MJS, Oliveira LR, Ilias M, Higa EFR. Community contributions to a FHU team work. Rev Latinoam Enferm 2007; 15:1065-71.

23. Crevelim MA, Peduzzi M. A participação da comunidade na equipe de saúde da família. Como estabelecer um projeto comum entre trabalhadores e usuários? Ciênc Saúde Coletiva 2005; 10:323-31.

24. Trad LAB, Esperidião MA. Gestão participativa e corresponsabilidade em saúde: limites e possibilidades no âmbito da Estratégia de Saúde da Família. Interface Comun Saúde Educ 2009; 13 Suppl 1:557-70.

25. Colomé ICS, Lima MADS, Davis R. Visão de enfermeiras sobre as articulações das ações de saúde entre profissionais de equipes de saúde da família. Rev Esc Enferm USP 2008; 42:256-61.

26. Kawata LS, Mishima SM, Chirelli MQ, Pereira MJB. O trabalho cotidiano da enfermeira na saúde da família: utilização de ferramentas da gestão. Texto \& Contexto Enferm 2009; 18:313-20.

27. Mishima SM. A gerência de serviços de atenção primária à saúde como instrumento para a reorganização da assistência à saúde - o caso do Programa de Saúde da Família [Tese de Livre-Docência]. Ribeirão Preto: Escola de Enfermagem de Ribeirão Preto, Universidade de São Paulo; 2003.

28. Shimizu HE, Rosales C. As práticas desenvolvidas no Programa Saúde da Família contribuem para transformar o modelo de atenção à saúde? Rev Bras Enferm 2009; 62:424-9.

29. Kell MCG, Shimizu HE. Existe trabalho em equipe no Programa Saúde da Família? Ciênc Saúde Coletiva 2010; 15 Suppl 1:1533-41.

30. Schwartz TD, Ferreira JTB, Maciel ELN, Lima RCD. Estratégia Saúde da Família: avaliando o acesso ao SUS a partir da percepção dos usuários da Unidade de Saúde de Resistência, na região de São Pedro, no município de Vitória (ES). Ciênc Saúde Coletiva 2010; 15:2145-54. 
31. Silva AS, Laprega MR. Avaliação crítica do Sistema de Informação da Atenção Básica (SIAB) e de sua implantação na região de Ribeirão Preto, São Paulo, Brasil. Cad. Saúde Pública 2005; 21:1821-8.

32. Schimith MD, Lima MADS. Acolhimento e vínculo em uma equipe do Programa Saúde da Família. Cad Saúde Pública 2004; 20:1487-94.

33. Silva KL, Rodrigues AT. Ações intersetoriais para promoção da saúde na Estratégia Saúde da Família: experiências, desafios e possibilidades. Rev Bras Enferm 2010; 63:762-9.

34. Ministério da Saúde. Avaliação para melhoria da qualificação da Estratégia Saúde da Família: resumo executivo. Brasília: Ministério da Saúde; 2005.

35. Departament of Health, Office of Public Health Practice. Community health assessment and action planning handbook (CHAAP) 2005-2009. Minnesota: Department of Health, Office of Public Health Practice; 2007.

36. Sarti TD. Avaliação da Estratégia Saúde da Família no Espírito Santo utilizando o instrumento de Avaliação para Melhoria da Qualidade da Estratégia Saúde da Família (AMQ) [Dissertação de Mestrado]. Vitória: Programa de Pós-Graduação em Saúde Coletiva, Universidade Federal do Espírito Santo; 2004.

37. Alves VS, Nunes M. O. Educação em saúde na atenção médica ao paciente com hipertensão arterial no Programa Saúde da Família. Interface Comun Saúde Educ 2006; 10:131-47.
38. Machado CV, Lima LD, Viana LS. Configuração da atenção básica e do Programa Saúde da Família em grandes municípios do Rio de Janeiro, Brasil. Cad Saúde Pública 2008; 24 Suppl 1:S42-57.

39. Escorel S, Giovanella L, Mendonça MH, Magalhães R, Senna MCM. Avaliação da implementação do Programa Saúde da Família em dez grandes centros urbanos: síntese dos principais resultados. Brasília: Ministério da Saúde; 2002.

40. Caetano R, Dain S. O Programa Saúde da Família e a reestruturação da atenção básica à saúde nos grandes centros urbanos: velhos problemas, novos desafios. Physis (Rio J.) 2002; 12:11-21.

41. Campos FE, Aguiar RAT, Oliveira VB. O desafio da expansão do Programa de Saúde da Família nas grandes capitais brasileiras. Physis (Rio J.) 2002; 12:47-58.

42. Sousa MF, Hamann EM. Programa Saúde da Família no Brasil: uma agenda incompleta? Ciênc Saúde Coletiva 2009; 14 Suppl 1:1325-35.

43. Rothman K, Greenland S, Lash T. Modern epidemiology. $3^{\text {rd }}$ Ed. Philadelphia: Lippincott Williams \&Wilkins; 2008

44. Ministério da Saúde. Política Nacional de Ciência, Tecnologia e Inovação em Saúde. Brasília: Ministério da Saúde; 2008.

Recebido em 02/Ago/2010

Versão final reapresentada em 07/Dez/2011 Aprovado em 12/Dez/2011 